\title{
VARIAÇÃO DAS ZOOXANTELAS E BRANQUEAMENTO NO HIDRÓIDE CALCÁRIO Millepora alcicornis LINNAEUS, 1758 NOS RECIFES DE PORTO DE GALINHAS - NORDESTE BRASILEIRO
}

\author{
Felipe Rafael Brasiliano CAVALCANTE ${ }^{1}$ \\ Fernanda Duarte AMARAL ${ }^{1}$
}

Recebido em: 17/09/2014

Aceito em: 20/10/2014

\section{RESUMO}

O presente estudo teve como objetivo analisar o padrão comportamental das zooxantelas associadas ao hidróide calcário Millepora alcicornis dos ambientes recifais da Praia de Porto de Galinhas - PE e determinar o grau de branqueamento sofrido por essa espécie e sua possível relação com a temperatura. A área estudada, Praia de Porto de Galinhas, Estado de Pernambuco, está situada entre as coordenadas $08^{\circ} 59^{\prime} 00^{\prime \prime}$ a $8^{\circ} 33^{\prime} 33^{\prime \prime}$ de latitude S e $35^{\circ} 00^{\prime} 27^{\prime \prime}$ a $34^{\circ} 59^{\prime} 00^{\prime \prime}$ de longitude $W$ uma das praias mais visitadas do litoral brasileiro, trazendo várias importâncias para a sociedade que dependem direta e indiretamente desse ambiente. Coletas mensais foram realizadas entre setembro de 2010 e setembro de 2011, através de mergulhos em apneia. Foram retirados quatro fragmentos por mês, totalizando 52; posteriormente processados e analisados no Laboratório de Ambientes Recifais (LAR/ UFRPE). A densidade populacional das zooxantelas de $M$. alcicornis apresentou uma média anual de $1,2 \times 10^{6}$ células $/ \mathrm{cm}^{2}$. 0 índice mitótico apresentou uma média anual de 6,2\% e o diâmetro celular, uma média anual de $11,75 \mu \mathrm{m}$. A correlação entre a densidade populacional e as zooxantelas foi significativamente alta, evidenciando a estreita ligação entre as variáveis. Além de perceber a variação das zooxantelas durante o período estudado, foi possível observar um evento de branqueamento, juntamente com o aparecimento de manchas brancas ocasionadas possivelmente pela doença White Pox.

Palavras chave: Simbiose, Coral-de-fogo, Ambientes recifais.

\section{ABSTRACT}

The present study aimed to analyze the behavioral patterns of zooxanthellae associated to calcareous hydroid Millepora alcicornis on reef environments of the Porto de Galinhas (PE) beach and determine the degree of bleaching undergone by this species and your possible relation with temperature. The studied area, Porto de Galinhas, Pernambuco State, is located between the coordinates $08^{\circ} 59^{\prime} 00$ "to 8 - 33'33" S latitude and $35^{\circ} 00^{\prime} 27$ " to $34^{\circ} 59^{\prime} 00^{\prime \prime}$ $W$ longitude, being one of the most visited beaches of the brazilian coast, bringing various importances to society who depend directly and indirectly this environment. Monthly samples were collected between September 2010 and September 2011, by diving in apnea. Four fragments were removed per month, totaling
52 fragments obtained and subsequently processed and analyzed in the Laboratório de Ambientes Recifais (LAR / UFRPE). The population density of zooxanthellae of $M$. alcicornis presented an annual average of $1.2 \mathrm{x}$ $10^{6}$ cells $/ \mathrm{cm}^{2}$. The mitotic index showed an annual average of $6.2 \%$ and the cell diameter, an annual average of $11.75 \mu \mathrm{m}$. The correlation between population density and the zooxanthellae was significantly high, indicating a close relationship between the variables. Besides the study understand the variation of zooxanthellae during the study period was also able to observe a bleaching event, along with the appearance of white spots caused possibly by White Pox disease.

Keywords: Symbiosis, Fire coral, Reef environment.

\section{INTRODUÇÃO}

As zooxantelas são endossimbiontes que se associam aos tecidos dos corais, hidróides calcários e outros invertebrados marinhos, de cor esverdeada/amarronzada que dão pigmentação e fornecem metabólitos fotossintéticos para alimentação e sobrevivência desses cnidários

\footnotetext{
${ }^{1}$ Departamento de Oceanografia. Universidade Federal de Pernambuco. E-mail: felipe_rbc@ibest.com.br

${ }^{2}$ Departamento de Biologia. Universidade Federal Rural de Pernambuco. E-mail: fdamaral@db.ufpe.br
} 
CAVALCANTE, F. R. B.; AMARAL, F. D. Variação das zooxantelas e branqueamento no hidróide calcário Millepora alcicornis Linnaeus, 1758 nos recifes de porto de galinhas - nordeste brasileiro.

(TRENCH,1971; STAT et al., 2006). Vários autores ainda afirmam que o processo de fotossíntese responsável pela produção de compostos orgânicos e oxigênio, é utilizado como fonte de energia para o crescimento do esqueleto dos corais e hidróides calcários (MUSCATINE, 1990; GATTUSO et al., 1999).

Várias atividades realizadas pelas zooxantelas nos corais envolvendo a utilização de carbono acontecem para manutenção, crescimento e fotossíntese (produção de carboidratos e liberação de oxigênio) das mesmas. O excesso da produção é doado ao hospedeiro para que auxilie no seu crescimento, reprodução e respiração celular, sendo também fonte de energia para a atividade de rejeição de sedimentos indesejáveis (FALKOWISK et al., 1984; MUSCATINE, 1990; GATTUSO et al., 1999; FURLA, 2000). Porém, entre as zooxantelas e os corais existe uma relação susceptível a variações, que depende de fatores externos, como, por exemplo: a mudança da temperatura, pH, salinidade, entre outros (PORTER, 1976; FITT et al., 2000; COSTA et al., 2001).

Muitos impactos naturais e humanos que ocorrem no sul do Atlântico são classificados em sobrepesca, sedimentação, atividades turísticas, desmatamento e desenvolvimento urbano, dentre outros. Entre os impactos naturais (muitas vezes também ligados ao homem), os mais importantes estão a proliferação de algas, aquecimento global, eventos de El Niño e branqueamento de corais (WILKINSON, 2008).

O fenômeno pantropical de branqueamento dos corais é caracterizado pela perda das zooxantelas e/ou clorofila nos tecidos dos corais, o que indica a ausência da coloração desses cnidários. (GLYNN, 1993; COSTA et al., 2001, SPALDING et al., 2001). Dentre os principais fatores do branqueamento, está o aumento da temperatura, que vem afetando os corais e hidróides calcários nas últimas décadas ao redor do mundo e no Brasil, influenciando também, principalmente no aumento de ácido carbônico na água, caracterizando uma acidificação e dificultando a taxa de calcificação nos seres que vivem nos oceanos (BRUNO et al., 2001; AMARAL et al., 2006b; COX, 2007; SEBÁSTIAN et al., 2009; VERON et al., 2009; WEIS, 2010).

Geralmente dentre os cnidários que mais sofrem estão os hidrocorais do gênero Millepora, pois são provavelmente mais sensíveis do que os corais escleractínios, e mais susceptíveis à morte após o branqueamento (GLYNN; WEERDT, 1991; MARSHALL; BAIRD, 2000). Espécimes do gênero Millepora têm uma distribuição circuntropical, localizada geralmente em regiões de águas mais rasas e quentes, porém, existe registro da expansão da área de ocorrência provavelmente devido aos eventos de mudanças climáticas (CLEMENTE et al. 2011).

Nos recifes brasileiros, um dos hidróides calcários mais comuns e importantes é a espécie Millepora alcicornis, sendo conhecida como "coral-de- fogo". Fatores externos (perturbações nos recifes) podem desencadear um desequilíbrio ecológico grande, afetando os seres que vivem sob sua proteção (BELÉM et al., 1986; PITOMBO et al., 1988; LEÃO; KIKUCHI, 2000; OLIVEIRA et al., 2008; GARCIA et al., 2009). Várias pesquisas com Millepora alcicornis no Brasil foram realizadas, contudo são pouco voltadas à observação da simbiose das zooxantelas ou variações sofridas por esses simbiontes devido à mudanças (AMARAL; COSTA, 1998; AMARAL et al. (2006, 2007, 2008; AMORIM, 2009; GARCIA et al., 2009).

Portanto, o presente trabalho visou estudar as zooxantelas presentes nos tecidos de Millepora alcicornis e foi capaz de observar o fenômeno do branqueamento do hidróide calcário, apresentando estudos ecológicos sobre a relação simbiótica existente entre esses organismos nos ambientes recifais da Praia de Porto de Galinhas - Pernambuco.

\section{ÁREA ESTUDADA}

A Praia de Porto de Galinhas (Fig. 1), está situada entre as coordenadas $08^{\circ} 59^{\prime} 00^{\prime \prime}$ a $8^{\circ} 33^{\prime} 33^{\prime \prime} \mathrm{S}$ e $35^{\circ} 00^{\prime} 27^{\prime \prime}$ a $34^{\circ} 59^{\prime} 00^{\prime \prime} W$. Encontra-se no Município de Ipojuca, aproximadamente a $65 \mathrm{Km}$ da cidade do Recife, sendo uma das praias mais visitadas do litoral brasileiro. Possui uma extensão com cerca de $5 \mathrm{Km}$ e essa praia possui ambientes recifais com diversos organismos, desde protozoários até cordados em geral, nas piscinas naturais de águas claras e quentes, que são visitados por pessoas do mundo inteiro, sendo um dos locais turísticos mais visitados no litoral do Estado de Pernambuco (BARRADAS et al., 2010; CPRH, 2011). 
CAVALCANTE, F. R. B.; AMARAL, F. D. Variação das zooxantelas e branqueamento no hidróide calcário Millepora alcicornis Linnaeus, 1758 nos recifes de porto de galinhas - nordeste brasileiro.

\section{MATERIAL E MÉTODOS}

As coletas foram realizadas nos meses de setembro de 2010 até setembro de 2011, através de mergulhos em apneia. Como as colônias não variaram muito em relação à batimetria, foram analisadas espécimes de 0 a $2 \mathrm{~m}$ de profundidade, na crista recifal, tendo sido anotadas várias informações sobre esses hidróides calcários em campo além das aferições da temperatura da água-do-mar, com o auxílio de um termômetro de mercúrio.

Foram retiradas quatro amostras por coleta, com cerca de $5 \mathrm{~cm}$ de tamanho em todos os meses de estudo, totalizando 52 fragmentos obtidos e analisados. Para a coleta dos fragmentos desses cnidários foram utilizados martelo e ponteira, posteriormente, esses foram armazenados individualmente em potes plásticos transparentes e com água do mar e informações necessárias para catalogá-las. As amostras foram retiradas com dois metros de distância uma da outra, para evitar retirada dos fragmentos de clones seguindo a metodologia apropriada (AMARAL et al., 1997; COSTA et al., 2001; AMORIM,2009).

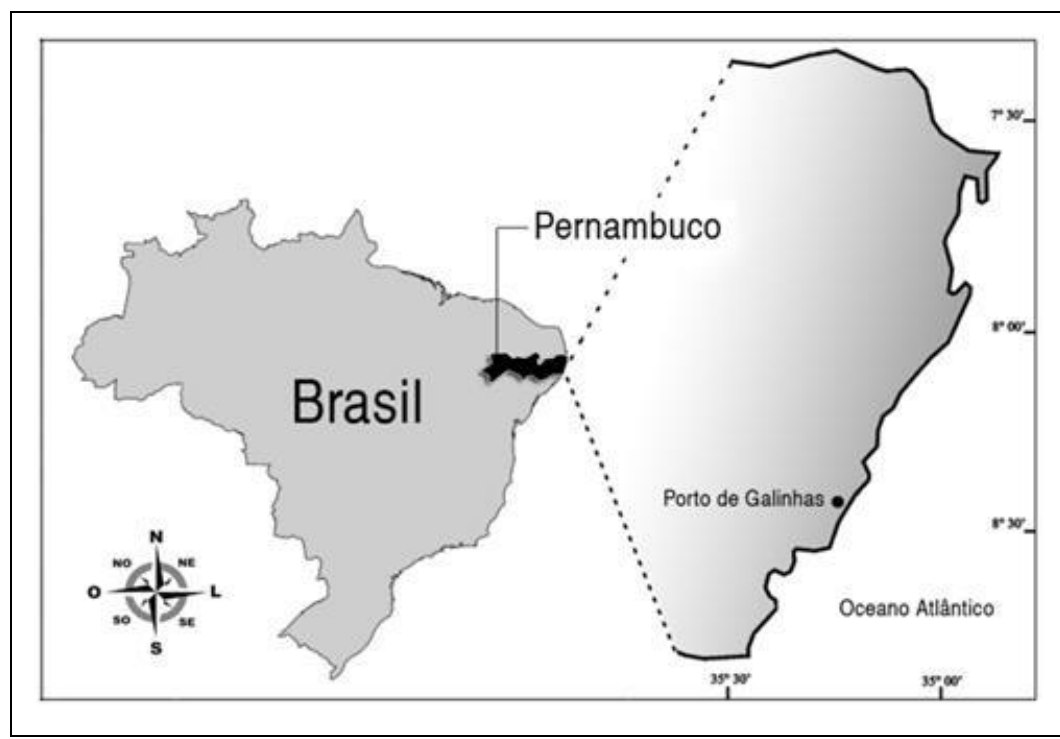

Figura 1 - Praia de Porto de Galinhas, litoral Sul de Pernambuco, Brasil

Os fragmentos foram processados e analisados no Laboratório de Ambientes Recifais (LAR/ UFRPE), onde foram retiradas as amostras de tecido através de jatos de água do mar filtrada (filtro Millipore Whatman, GF/ C - fibra de vidro de $47 \mathrm{~mm}$ diâmetro) provenientes do equipamento de extração Waterpik (Fig. 5). Logo após a retirada do tecido, as amostras foram fixadas com Lugol. As amostras extraídas foram armazenadas em potes pequenos com as informações de quantidade de água do mar utilizada para retirar o tecido do hidróide, mês de coleta e o número da amostra.

A análise do diâmetro das zooxantelas foi feita a partir de ocular micrométrica do microscópio (Bel Photonics) e, para estimar a densidade populacional foi utilizada a lâmina de Fuchs-Rosenthal, metodologia seguida por Carricart-Ganivet; Beltrán-Torres (1993), Costa et al. (2008) e Amorim (2009). As áreas dos fragmentos foram mensuradas a partir da Regressão Linear e Correlação de Pearson, através do peso das folhas de alumínio enroladas aos fragmentos dos hidróides calcários, seguindo Amorim (2009). O número de zooxantelas encontradas foi convertido em número de células $\times 10^{6} / \mathrm{cm}^{2}$, o índice mitótico foi calculado em porcentagem (\%) e o diâmetro celular foi observado em micrômetros $(\mu \mathrm{m})$.

$\mathrm{Na}$ análise dos dados foi utilizado o programa STATISTICA 7.0 com índice de significância de $5 \%$. Para a análise entre os meses da densidade populacional utilizou-se a ANOVA (One way) e para o índice mitótico e diâmetro celular o Kruskal- Wallis. Os dados de sazonalidade foram analisados a partir da análise estatística de Mann- Whitney. A correlação linear de Pearson foi utilizada para avaliar a relação entre a densidade populacional de zooxantelas e a temperatura superficial da água-do-mar. 
CAVALCANTE, F. R. B.; AMARAL, F. D. Variação das zooxantelas e branqueamento no hidróide calcário Millepora alcicornis Linnaeus, 1758 nos recifes de porto de galinhas - nordeste brasileiro.

\section{RESULTS/RESULTADOS}

A densidade populacional das zooxantelas apresentou uma média anual de $1,2 \times 10^{6}$ células/ $\mathrm{cm}^{2}$, com as maiores médias nos meses de setembro de 2010 e julho de $2011(1,6 \times$ $10^{6}$ células $\left(\mathrm{cm}^{2}\right)$. O índice mitótico apresentou uma média anual de $6,2 \%$, com maior valor para o mês de setembro de $2010(11 \%)$ e o menor no mês de agosto de $2011(1,5 \%)$. O diâmetro celular apresentou uma média anual de $11,75 \mu \mathrm{m}$ com valores maiores para o mês de fevereiro de $2011(13,13 \mu \mathrm{m})$ e o menor no mês de agosto de $2011(10,44 \mu \mathrm{m})$. Todas as três variáveis tiveram diferenças significativas entre os meses (Fig. 2, p<0,05).

Foi registrado também branqueamento das colônias durante o período estudado. Tal fenômeno foi observado nos meses de março e abril de 2011 (Fig. 3), onde foi percebido menores valores para a densidade populacional. A partir de maio as colônias foram observadas sadias sem haver sinais de branqueamento e com um aumento na densidade populacional. No início do período crítico de branqueamento foram visualizadas manchas brancas inicialmente no mês de fevereiro de 2011, onde pode ter sido ocasionada por "White Pox", e que também foram visualizados mais focos para os meses de junho e julho de 2011.

A temperatura (Fig. 4) teve uma média anual de $28,46^{\circ} \mathrm{C} \pm 1,46 \mathrm{com}$ máximo de $30^{\circ} \mathrm{C}$ (período antecedente ao branqueamento) e mínimo de $26^{\circ} \mathrm{C}$. A correlação entre a densidade populacional das zooxantelas e a temperatura mostrou ser significativamente alta e negativa $(r=-0,88, p<0,05)$, evidenciando uma interação muito forte entre as variáveis analisadas.

Em relação à sazonalidade, a densidade populacional teve diferenças significativas entre os períodos seco e chuvoso (Mann- Whitney $\mathrm{p}<0,05$ ), sendo o período chuvoso com maiores valores (Fig. 5). Foram encontradas diferenças significativas de sazonalidade também para índice mitótico e diâmetro celular (Mann- Whitney $\mathrm{p}<0,05$ ), com maiores valores obtidos no período seco para ambos (Fig. 6 e 7).

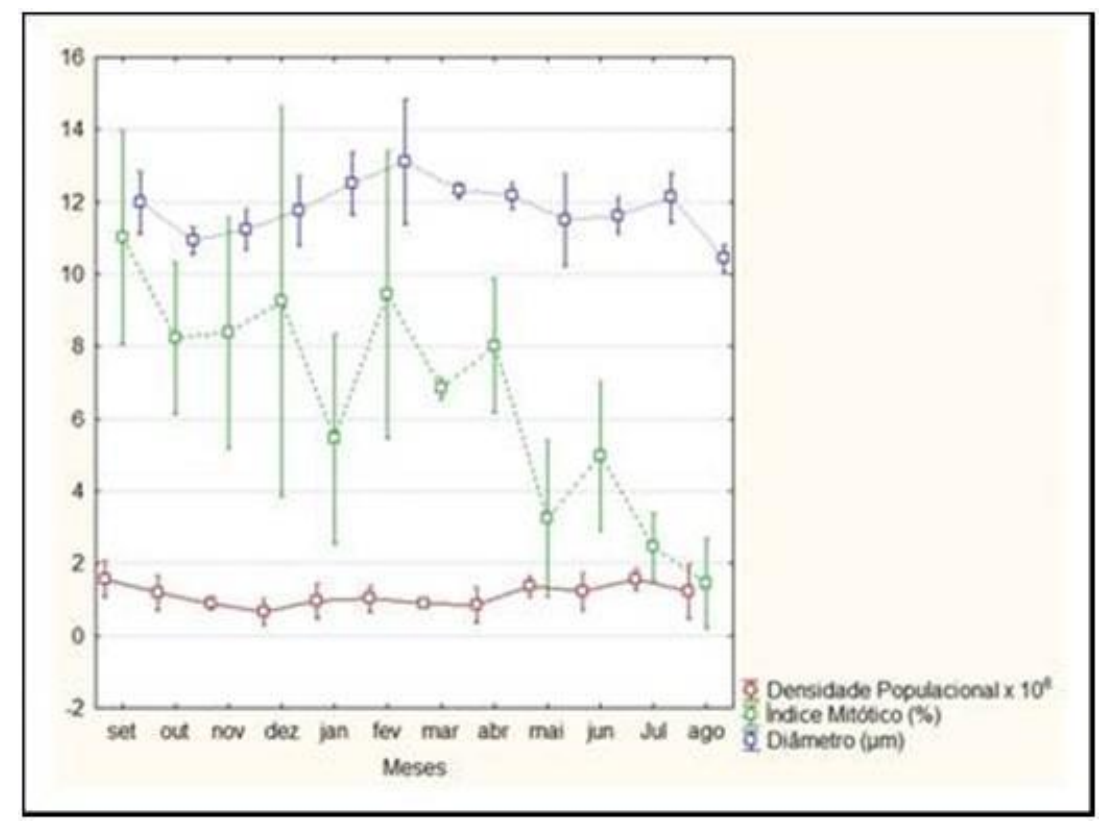

Figura 2 - Média e desvio padrão (barras verticais) da densidade populacional ( $\times 10^{6}$ células/ $\left.\mathrm{cm}^{2}\right)$, índice mitótico (\%) e diâmetro celular $(\mu \mathrm{m})$ das zooxantelas em Millepora alcicornis durante setembro de 2010 a agosto de 2011, na praia de Porto de Galinhas (PE). 
CAVALCANTE, F. R. B.; AMARAL, F. D. Variação das zooxantelas e branqueamento no hidróide calcário Millepora alcicornis Linnaeus, 1758 nos recifes de porto de galinhas - nordeste brasileiro.

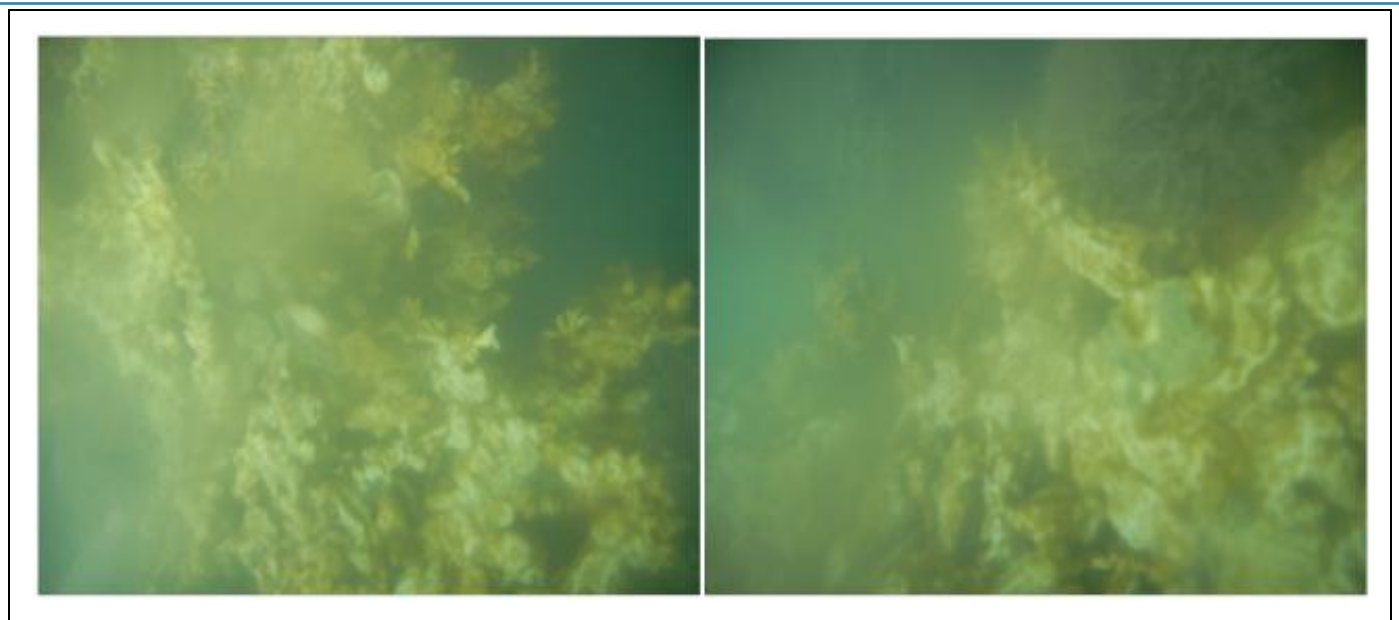

Figura 3 - Colônias branqueadas de M. alcicornis nos ambientes recifais da Praia de Porto de Galinhas (PE) em março de 2011.

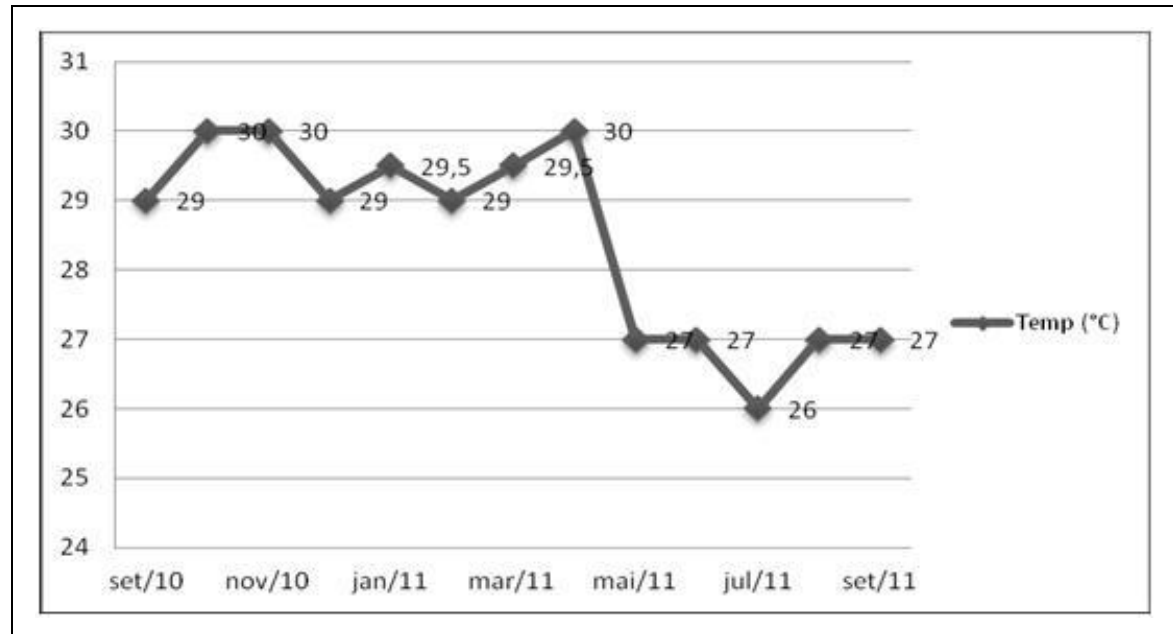

Figura 4 - Temperatura da água-do-mar durante setembro de 2010 a agosto de 2011, na praia de Porto de Galinhas (PE).

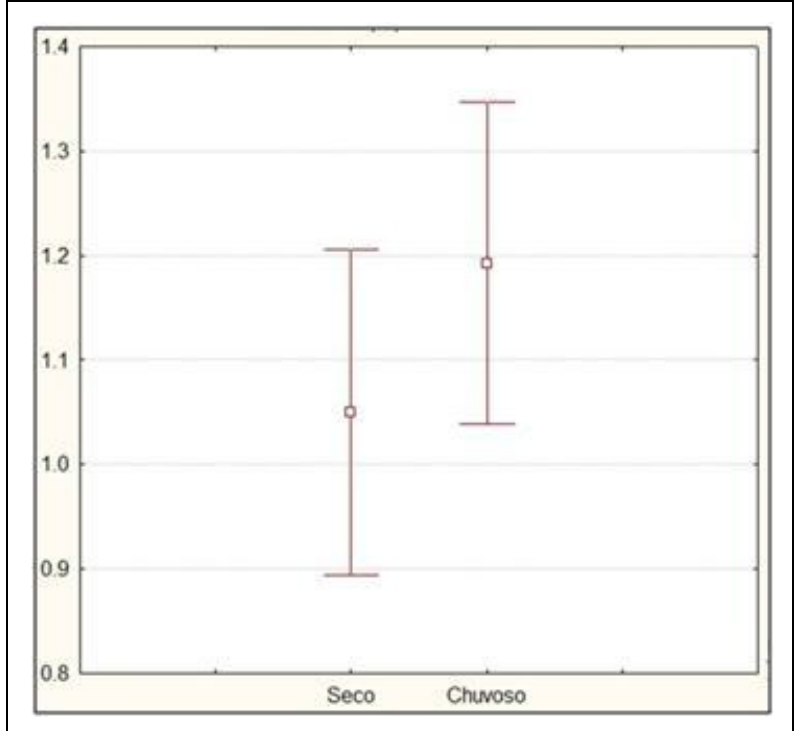

Figura 5 - Densidade populacional $\left(\times 10^{6}\right.$ células $/ \mathrm{cm}^{2}$ ) das zooxantelas em Millepora alcicornis durante o período seco e chuvoso (2010/2011), na praia de Porto de Galinhas (PE) 
CAVALCANTE, F. R. B.; AMARAL, F. D. Variação das zooxantelas e branqueamento no hidróide calcário Millepora alcicornis Linnaeus, 1758 nos recifes de porto de galinhas - nordeste brasileiro.

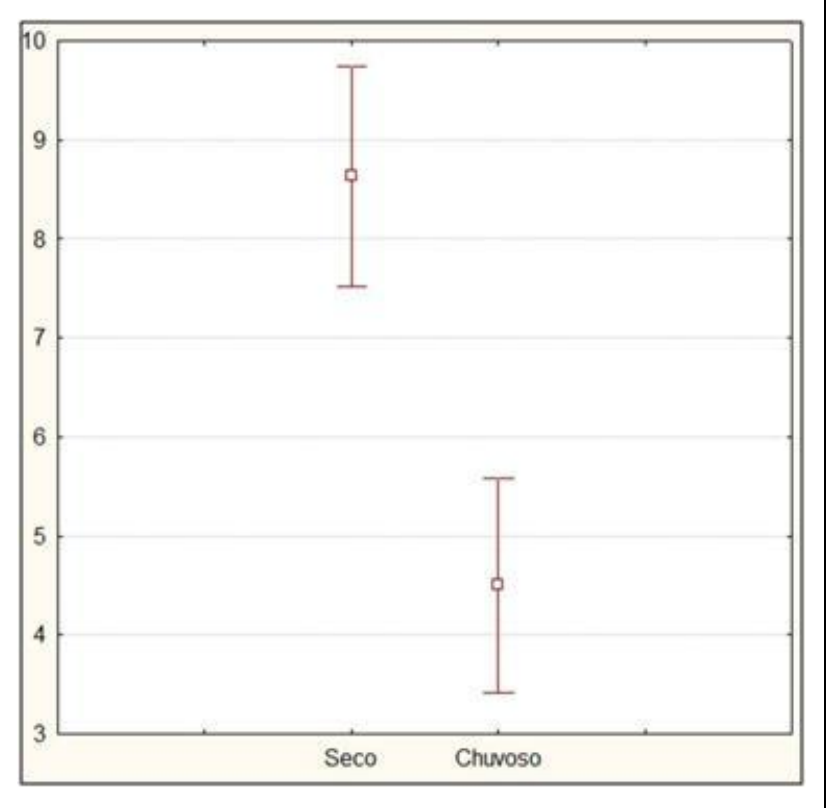

Figura 6 - Índice mitótico (\%) das zooxantelas em Millepora alcicornis durante o período seco e chuvoso (2010/2011), na praia de Porto de Galinhas (PE).

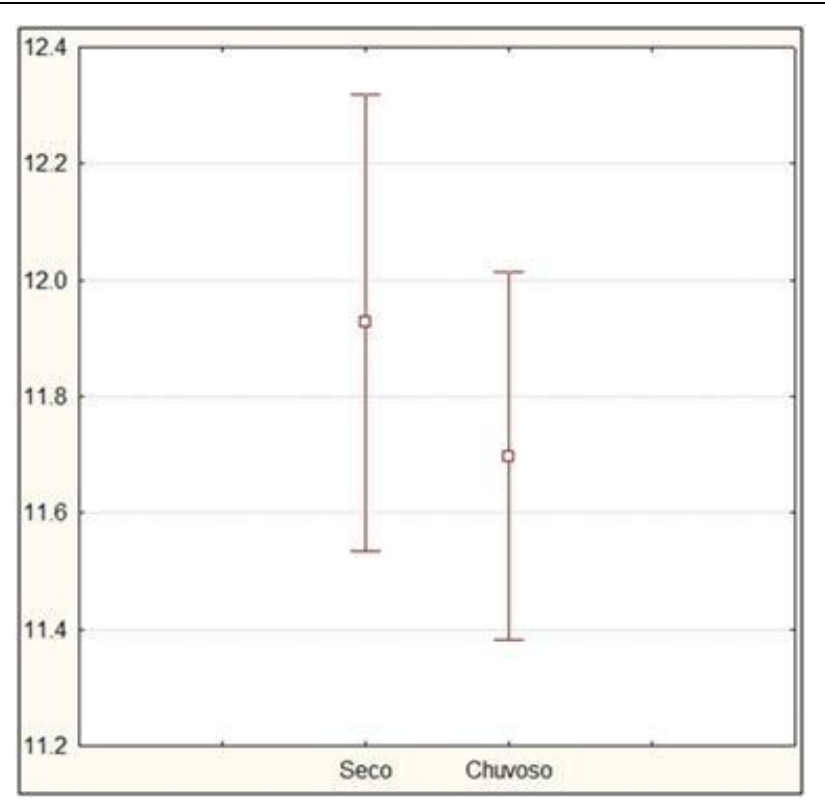

Figura 7 - Diâmetro celular $(\mu \mathrm{m})$ das zooxantelas em Millepora alcicornis durante o período seco e chuvoso (2010/2011), na praia de Porto de Galinhas (PE).

\section{DISCUSSÃO}

O presente estudo mostrou que a variação de densidade populacional é significativamente diferente entre os meses, corroborando com os resultados de Amorim (2009), que trabalhando com as zooxantelas dessa mesma espécie nos recifes de Picãozinho (Paraíba) verificou diferentes médias para um mesmo mês de coleta. Isso reforça a observação de que as mudanças na densidade não dependem somente de fatores externos, mas da fisiologia dos organismos que estão interagindo, podendo-se admitir que a $M$. alcicornis é uma espécie instável em relação à quantidade de zooxantelas presentes em seu tecido. Ainda em relação à mesma variável, a referida autora constatou que a média anual das zooxantelas foi de 1,02 $\mathrm{x}$ $10^{6}$ células $/ \mathrm{cm}^{2}$ semelhante a encontrada na referida pesquisa que foi de $1,2 \times 10^{6} \mathrm{células} / \mathrm{cm}^{2}$. Mesmo assim, a média encontrada é mais baixa da que foi encontrada por Amaral; Costa (1998) que registrou $1,44 \times 10^{6}$ células $/ \mathrm{cm}^{2}$ para as zooxantelas de $M$. alcicornis dos recifes de Tamandaré - PE.

Ainda em relação à densidade populacional das zooxantelas em M. alcicornis, a diferença significativa ocorrida entre os meses mostra que essa espécie possui flutuações mensais em sua 
CAVALCANTE, F. R. B.; AMARAL, F. D. Variação das zooxantelas e branqueamento no hidróide calcário Millepora alcicornis Linnaeus, 1758 nos recifes de porto de galinhas - nordeste brasileiro.

quantidade de simbiontes. Esse resultado, é semelhante ao observado por Costa et al. (2004), que realizando estudos sobre zooxantelas no coral escleractínio Montastraea cavernosa Linnaeus, 1767 observaram uma variação significativa entre os meses. Além disso, o trabalho mencionado anteriormente registrou uma quantidade de zooxantelas para esses corais bem maiores do que as encontradas no presente estudo para o hidróide calcário pesquisado.

A densidade populacional na estação chuvosa obteve resultados maiores que a estação seca, contudo o trabalho de Amorim (2009) relata que os meses chuvosos foram os que tiveram menores densidades e contrastam com resultados obtidos pelo estudo atual. Eloy (2005), com estudos realizados em zoantídeo Palythoa caribaeorum Duchassaing \& Michelotti, 1860, relatou que esse aumento na densidade populacional pode ser devido ao maior esforço para que haja captação de luz pelas zooxantelas. Contudo, os dados de diâmetro celular mostraram ser maiores, quando a densidade populacional era mais baixa, isso evidencia uma possível estratégia para manter as condições ideais na colônia. Em relação ao índice mitótico, a estação seca mostrou uma maior quantidade de divisões celulares podendo essa ser provavelmente uma estratégia de sobrevivência desses dinoflagelados ou uma adaptação, pois este fato ocorreu no período que houve o fenômeno de branqueamento.

Os resultados observados para o diâmetro mostraram que os maiores diâmetros foram observados na estação seca o que ajuda na maior captação de luz solar. Amaral; Costa (1998) evidenciaram um diâmetro maior em zooxantelas de Millepora alcicornis (12,5 $\mu \mathrm{m})$ enquanto no presente estudo foi registrado valores menores $(11,75 \mu \mathrm{m})$. Quando comparadas à outras espécies de corais escleractínios como Favia gravida e Siderastrea stellata as referidas autoras evidenciaram como diâmetro maior para zooxantelas de Millepora alcicornis, contudo suas medições foram menores que as da espécie do hidróide calcário Millepora braziliensis.

Fagoonee et al. (1999) relataram que as flutuações das populações das zooxantelas nos tecidos dos corais são resultantes dos eventos de branqueamento e promovem variações sazonais. Os autores mostraram que em eventos de branqueamento houve altas flutuações na densidade populacional entre os anos de estudo e que devido a isso se criam ciclos. A pesquisa atual corrobora com essa ideia e revelou que esse branqueamento sofrido nos meses da estação seca promoveu uma variação na densidade populacional, uma diminuição na quantidade de simbiontes nos corais. Além disso, Poggio et al. (2009) evidenciaram o branqueamento sazonal para colônias de Siderastrea spp. em poças recifais juntamente com o menor valor de densidade populacional para os meses mais secos.

Os estudos realizados por Fitt et al. (2000) mostraram resultados semelhantes ao presente trabalho em relação às diferenças sazonais com corais escleractínios, pois as espécies analisadas - Acropora palmata, A. cervicornis, Montrastraea faveolata, M. annularis apresentaram padrões sazonais com dados menores de densidade populacional nos períodos de verão e de branqueamento. Fitt et al. (2001) pontuam também que os corais estão mais susceptíveis a um maior densidade de zooxantelas presentes nos tecidos nos meses de menor incidência e de menor quantidade de radiação solar, e nos meses de maior incidência de luz possuem uma quantidade menor de zooxantelas associadas aos corais devido à um excesso de radiação luminosa.

De acordo com Lewis (1989), o branqueamento observado em indivíduos do gênero Millepora é avaliado como rápido e eles possuem um período de curta duração, recuperando-se mais facilmente em eventos de menor estresse. Porém, de acordo com Glynn et al. (1991), se o evento for de alta duração, eles podem chegar à morte mais rápido que os corais escleractínios. Possivelmente devido ao fato que o evento mostrado ser de curta duração, não foi observado anteriormente na costa pernambucana.

Esse desequilíbrio gerado pela diminuição da população de zooxantelas causa um enfraquecimento desses organismos e os tornam mais susceptíveis ao branqueamento e a doenças em corais. Isso é observado no trabalho de Fitt et al. (2001) que atribui causas da doença a períodos de perturbações que os corais e outros organismos marinhos sofrem. De acordo com Rowan et al. (1997), o branqueamento está relacionado com a constituição genética (padrão x genético) das zooxantelas, porém Brown (1997) e Hoegh-Guldberg (1999) afirmam que a forma do crescimento da colônia aliada à espessura dos tecidos dos corais pode 
CAVALCANTE, F. R. B.; AMARAL, F. D. Variação das zooxantelas e branqueamento no hidróide calcário Millepora alcicornis Linnaeus, 1758 nos recifes de porto de galinhas - nordeste brasileiro.

influenciar na incidência de branqueamento. Corroborando com os estudos anteriores, Loya et al. (2001) demonstraram que os corais de menor porte preferencialmente conseguem resistir e sobreviver aos eventos de branqueamento.

O branqueamento de indivíduos de $M$. alcicornis foi registrado para a costa brasileira por Amaral et al. (2006), Leão et al. (2008) e Amorim (2009). Amaral et al. (2006) publicaram um artigo informando sobre o branqueamento sofrido pelos indivíduos do gênero Millepora no Parque Estadual Marinho do Parcel do Manuel Luiz, dentre esses, colônias de M. alcicornis e a espécie recém descoberta M. laboreli Amaral in Amaral et al., 2008. Posteriormente, Leão et al. (2008) também informaram sobre eventos que influenciaram no branqueamento desse hidróide calcário para os recifes da Bahia, e sobre os períodos curtos de branqueamento dessa espécie. Para Pernambuco, somente Barradas et al. (2010) observou tal fenômeno para alguns indivíduos da espécie em questão em 2004, sendo este o segundo registro do branqueamento nesses indivíduos, para a praia Porto de Galinhas.

No início do período crítico de branqueamento foram visualizadas manchas brancas inicialmente no mês de fevereiro de 2011, onde pode ter sido ocasionada por "White Pox", que de acordo com Patterson et al. (2002), uma doença causada em corais que apresenta-se com manchas brancas na superfície das colônias. Amorim (2009) e Amorim et al. (2011) também encontraram pontos brancos, com formatos circulares e multifocais nos tecidos dos hidróides calcários da espécie M. alcicornis e caracterizaram como "White Pox".

Além dessas observações anteriores, foram visualizados mais focos também para os meses de junho e julho de 2011 (Fig. 7), quando houve o reaparecimento dessas manchas brancas em várias colônias, mostrando a sensibilidade desses ambientes a alterações no meio, possivelmente devido a uma grande carga microbiológica. Essa doença causada pela bactéria Serratia marcescens Bizio, 1823 está relacionada a perda de $80 \%$ da cobertura do coral escleractínio Acropora palmata do Santuário Marinho "Florida Keys" e no Caribe. De acordo com Patterson et al. (2002), ela é causada pela bactéria patogênica oportunista transmitida em águas quentes e associada ao trato urinário de humanos e outras infecções bacterianas. Isso leva a pensar que essa doença observada na Praia de Porto de Galinhas - PE pode estar relacionada com o aporte de esgotos clandestinos ou com a atividade turística nessa região.

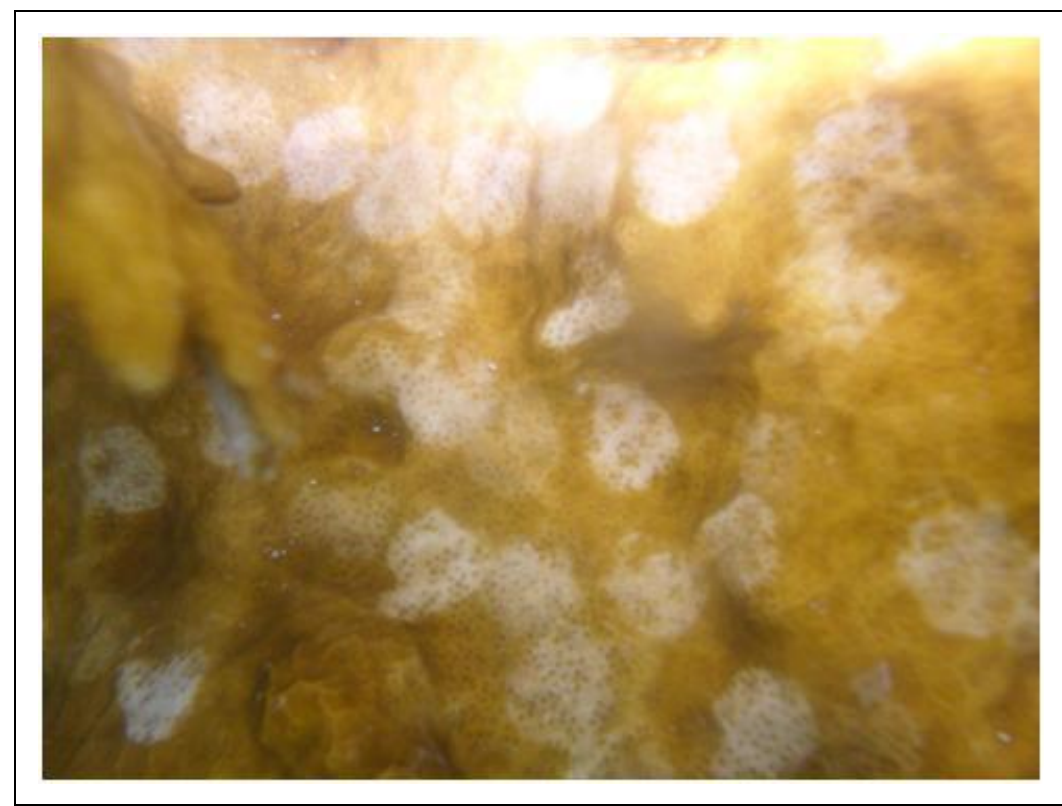

Figura 7 - Colônias de Millepora alcicornis dos ambientes recifais da Praia de Porto de Galinhas - PE com manchas brancas no mês de julho de 2011.

\section{CONCLUSÃO}

O presente estudo observou uma variação anual, em relação aos meses e estações entre o ano de 2010 e 2011 no padrão comportamental para as zooxantelas de Millepora alcicornis para a Praia de Porto de Galinhas - PE. Além disso, foi verificado um evento de branqueamento da referida espécie e possíveis casos da doença White Pox. Contudo, a falta de um plano efetivo de 
CAVALCANTE, F. R. B.; AMARAL, F. D. Variação das zooxantelas e branqueamento no hidróide calcário Millepora alcicornis Linnaeus, 1758 nos recifes de porto de galinhas - nordeste brasileiro.

monitoramento dos recifes pernambucanos, além de pouca difusão sobre o estudo da simbiose de zooxantelas dessa espécie dificulta em um maior conhecimento ecológico acerca desse hidrocoral que é tão fundamental para a construção dos recifes brasileiros.

\section{AGRADECIMENTOS}

Agradecemos à FACEPE pela concessão da Bolsa de Iniciação Científica (PIBIC/ FACEPE) do primeiro autor e à UFRPE pelo espaço concedido para as análises do estudo, em especial ao Laboratório de Ambientes Recifais (LAR/UFRPE), ao Departamento de Biologia pelo apoio, e à Aline Spíndola (Programa de Pós- Graduação em Parasitologia - UFPel) pela ajuda na confecção do manuscrito.

\section{REFERÊNCIAS BIBLIOGRÁFICAS}

AMARAL, F. D.; SILVA, R. S.; MAURÍCIO-DA-SILVA, L.; SOLÉ-CAVA, A. M. Molecular systematics of Millepora alcicornis Linnaeus, 1758 and M. braziliensis Verril, 1868 (Hydrozoa: Milleporidae) from Brazil. Proceedings of the 8th Internacional Coral Reef Symposium, $v$. 2, p. 1577-1580, 1997.

AMARAL, F. D.; COSTA, C. F. Zooxantelas dos hidrocorais Millepora alcicornis e Millepora braziliensis e dos corais Favia gravida e Siderastrea stellata de Pernambuco. Trabalho Oceanográfico, UFPE, v. 26, n. 1, p. 123 - 133, 1998.

AMARAL, F. D.; HUDSON, M.; STEINER, A. Q. Note on the widespread bleaching observed at the Manuel Marine State Park, Maranhão, Brazil. Arquivo de Ciências do Mar, v. 39, p.138-141, 2006b.

AMARAL, F. D.; HUDSON, M. M.; STEINER, A. Q.; RAMOS, C. A. C. Corals and calcified hydroids of the Manuel Luiz Marine State Park (State of Maranhão, Northeast Brazil). Biota Neotropica, vol.7, n. 3, p.73-81, 2007.

AMARAL, F. D.; STEINER, A. Q.; BROADHURST, M. K.; CAIRNS, S. D. Na overview of the shallow-water calcified hydroids from Brazil (Hydrozoa: Cnidaria), including the description of a new species. Zootaxa, p. 58-68, 2008.

AMORIM, T. P. de L. Microssimbiontes associados à Millepora alcicornis (Linnaeus, 1758) (Cnidaria, Hydrozoa) dos recifes costeiros de Picãozinho, João Pessoa - PB. Dissertação de Mestrado (Mestrado em Ciências Biológicas), João Pessoa.125f. 2009.

AMORIM, T. P. L; COSTA, C. F.; SASSI, R. Branqueamento e doenças em cnidários dos recifes costeiros de Picãozinho, Nordeste do Brasil. Tropical Ocenography, Recife, v. 40, n. 1, p. 185-201, 2011.

BARRADAS, J. M.; AMARAL, F. M. D. ; HERNANDEZ, M. I. M. ; FLORES, M. ; STEINER, A. Q. Spatial distribution of benthic macroorganisms on reef flats at Porto de Galinhas Beach (northeastern Brazil), with special focus on corals and calcified hydroids. Biotemas (UFSC), v. 23, p. 1-11. 2010.

BELÉM, M.J.C.; ROHLFS, C.; PIRES, D.O.; CASTRO, C. B.;YOUNG, P.S. S.O.S. Corais. Ciência Hoje, Sociedade Brasileira para o Progresso da Ciência, Rio de Janeiro, RJ, v. 5, p.35-42. 1986.

BROWN, B. E. Coral bleaching: causes and consequences. Coral Reefs, v. 16, p. 129-138, 1997.

BRUNO, J. F.; SIDDON, C. E.; WITMAN, J. D.; COLIN, P. L.; TOSCANO, M. A. El Niño Related Coral Bleaching in Palau, western Caroline Islands. Coral Reefs, v. 20, p.127-136. 2001.

CARRICART-GANIVET, J. P.; BELTRÁN-TORRES, A. V. Zooxanthellae and chlorophylla a responses in the scleractinian coral Montastraea cavernosa at Triângulos W Reef, Campeche Bank, México. Revista de Biologia Tropical, v.41, n.3, p.491-494, 1993.

CLEMENTE, S.; ROdRÍGUeZ, A.; BRITO, A.; RAMOS, A.; MONTERROSO, Ó; HERNÁNDEZ, J. C. On the occurrence of the hydrocoral Millepora (Hydrozoa: Milleporidae) in the subtropical eastern Atlantic (Canary Islands): is the colonization related to climatic events? Coral Reefs, $v$. 30, p. 237-240, 2011. 
CAVALCANTE, F. R. B.; AMARAL, F. D. Variação das zooxantelas e branqueamento no hidróide calcário Millepora alcicornis Linnaeus, 1758 nos recifes de porto de galinhas - nordeste brasileiro.

COSTA, C. F.; AMARAL F. D.; SASSI R. Branqueamento em Siderastrea stellata (Cnidaria, Scleractinia) da Praia de Gaibu - Pernambuco, Brasil. Revista Nordestina de Biologia. João Pessoa, v. 15, n. 1, p. 15-22, 2001.

COSTA, C. F.; AMARAL, F. M. D. Population density and photosynthetic pigment content in symbiotic dinoflagellates in the Brazilian scleractinian coral Montastraea cavernosa (Linnaeus, 1767). Revista Brasileira de Oceanografia, São Paulo, v. 52, n. 2, p. 93-99, 2004.

COSTA, C. F.; SASSI, R.; GORLACH-LIRA, K. Uma abordagem metodológica para o estudo das zooxantelas de corais do Brasil. Boletim do Laboratório de Hidrobiologia, v. 21, p. 83-94. 2008.

COX, E. F. Continuation of Sexual Reproduction in Montipora capitata Following Bleaching. Coral Reefs, 26, p.721-724, 2007.

$\mathrm{CPRH}$ - Agência Estadual de Meio Ambiente e Recursos hídricos. Balneabilidade nas praias de Pernambuco. Acessado em 22:00h de 06/12/2011.

ELOY, C. C. Estudo da microbiota simbionte de Palythoa caribaeorum (Duchassaing \& Michelotti, 1860) (Cnidaria - Zoantidea) da praia do Cabo Branco, Paraíba, Brasil, com ênfase nas zooxantelas. Dissertação de Mestrado (Mestrado em Ciências Biológicas). Universidade Federal da Paraíba. 79f, 2005.

FAGOONEE, I.; WILSON, H. B.; HASSELL, M. P.; TURNER, J. R. The Dynamics of Zooxanthellae Populations: A Long-Term Study in the Field. Science, v. 283, p. 843-845. 1999.

FALKOWSKI, P. G.; DUBINSKY, Z.; MUCATINE, L.; PORTER, J. W. Ligth and the bioenergetics of a symbiotic coral. Bioscience, v. 34, p. 705-709. 1984.

FITT, W.K., MCFARLAND, F.K., WARNER, M.E. \& CHILCOAT, G.C. Seasonal patterns of tissue biomass and densities of symbiotic dinoflagellates in reef corals and relation to coral bleaching. Limnology and Oceanography, v. 45, n. 3, p.677-685, 2000.

FITT, W. K.; BRONW, B. E.; WARNER, M. E.; DUNNE, R. P. Coral bleaching: interpretation of thermal tolerance limits and thermal thresholds in tropical corals. Coral Reefs, v. 20, p. 51-65. 2001.

FURLA, P.; GALGANI. I.; DURAND, I.; ALLEMAND, D. Sources and mechanisms of inorganic carbon transport for coral calcification and photosynthesis. Journal of Experimental Biology, v. 203, p. 3445-3457. 2000.

GARCIA, T. M; MATTHEWS-CASCON, H.; FRANKLIN-JUNIOR, W. Millepora alcicornis (Cnidaria: Hydrozoa) as substrate for benthic fauna. Brazilian Journal of Oceanography, v. 57, n. 2, p.153-155, 2009.

GATTUSO, J. P.; ALLEMAND, D.; FRANKIGNOULLE, M. Photossynthesis and calcification at cellular, organismal and community levels in coral reefs: A review on interactions and control of carbonate chemistry. American Zoologist, v. 39, p. 160-183. 1999.

GLYNN, P. W. Coral reef bleaching: Ecological perspectives. Coral Reefs, v.12, p.1-17. 1993.

GLYNN, P. W.; WEERDT, W. H. de. Elimination of two reef-building hydrocorals following the 1982-83 El Niño warming event. Science, v. 256, p.69-70. 1991.

HOEGH-GULDBERG, O. Climate change, coral bleaching and the future of the world's coral reefs. Marine \& Freshwater Research, v. 50, p. 839-866. 1999.

LEÃO, Z. M. A. N.; KIKUCHI, R. K. P. The Abrolhos reefs of Brazil. In: Seeliger, U. \& Kjerfve, B. (eds.) Coastal Marine Ecosystems of Latin America, p. 83- 96, Springer-Verlag, Ecological Studies 144, Berlin Heidelberg New York. 2000.

LEÃO, Z. M. A. N.; KIKUCHI, R. K. P.; OLIVEIRA, M. D. M. Branqueamento de corais nos recifes da Bahia e sua relação com eventos de anomalias térmicas nas águas superficiais do oceano.

Biota Neotropica, v.8, n. 3. 2008.

LEWIS, J. B. The ecology of Millepora: A review. Coral Reefs, v.8, n.3, p.99- 107, 1989. 
CAVALCANTE, F. R. B.; AMARAL, F. D. Variação das zooxantelas e branqueamento no hidróide calcário Millepora alcicornis Linnaeus, 1758 nos recifes de porto de galinhas - nordeste brasileiro.

LOYA, Y.; SAKAI, K.; YAMAZATO, K.; NAKAMO, Y. SAMBALL, H.; VAN WOESIK, R. Coral bleaching: the winners and the loosers. Ecology Letters, v. 4, p. 122-131, 2001.

MARSHALL, P. A.; BAIRD, A. H. Bleaching of corals on the Great Barrier Reef: differential susceptibilities among taxa. Coral Reefs, v.19, p.155-163. 2000.

MUSCATINE, L. The role of symbiontic algae in carbon and energy flux in reef corals. Coral Reefs. Amsterdam, p. 75-87. 1990.

OLIVEIRA, M. D. M.; LEÃO, Z. M. A. N; KIKUCHI, R. K. P. Cultivo de Millepora alcicornis como uma ferramenta para Restauração e Manejo dos Ecossistemas Recifais do Nordeste do Brasil.

Revista da Gestão Costeira Integrada, v.8, n.2, p.183-201. 2008.

PATTERSON, K. L.; PORTER, J. W.; RITCHIE, K. B.; POLSON, S. W.; MUELLER, E.; PETERS, E. C.; SANTAVY, D. L.; SMITH, G. W. The etiology of white pox a lethal disease of the Caribbean elkhorn coral Acropora palmata. Proceedings of the National Academy of Sciences of the United States of America (PNAS), v. 99, n. 13, p. 8725-8730, 2002.

PITOMBO, F., RATTO, C.C.; BELÉM M. J. C. Species diversity and zonation patter of hermatypic corals at two fringing reefs Abrolhos archipelago, Brazil. Proceedings of the 6th

International Coral Reef Symposium, v. 2, p. 817-820, 1988.

POGGIO, C.; LEÃO, Z.; MAFALDA-JÚNIOR, P. Registro de branqueamento sazonal em Siderastrea spp. em poças intermareais do recife de Guarajuba, Bahia, Brasil. Interciência, v. 34, n. 7, p. 502-506, 2009.

PORTER, J.W. Autotrophy, heterotrophy, and resource partitioning in Caribbean reef-building corals. The American Naturalist. vol. 110, n. 975, p.731, 1976.

ROWAN, R.; KNOWLTON, N.; BAKER, A.; JARA, J. Landscape ecology of algal symbionts creates variation in episodes of coral bleaching. Nature, v. 388, p. 265-269, 1997.

SEBASTIÁN, C. R.; SINK, K. J.; MCCLANAHAN, T. R.; COWAN, D. A. Bleaching response of corals and their Symbiodinium communities in Southern Africa. Marine Biology. v.156, p.2049-2062, 2009.

SPALDING, M. D.; RAVILIOUS, C.; GREEN, E. P. World Atlas of Coral Reefs. Prepared at the UNEP World Conservation Monitoring Centre. University of California Press, Berkeley, USA. 2001.

STAT, M.; CARTER, D.; HOEGH-GULDBERG, O. The evolutionary history of Symbiodinium and scleractinian hosts - symbiosis, diversity, and the effect of climate change. Perspectives in plant ecology, evolution and systematics, v. 8, n. 1, p. 23-43. 2006.

TRENCH, R. K. The physiology and biochemistry of zooxanthellae symbiotic with marine coelenterates. Proceedings of the Royal Society of London. Series b. 177: 225-235. 1971.

VERON, J.E.N.; HOEGH - GULDBERG, O.; LENTON, T. M.; LOUGH, J. M.; OBURA, D. O.; PEARCE - KELLY, P.; SHEPPARD, C. R. C.; SPALDING, M.; STAFFOR - SMITH, M. G.; ROGERS A. D. The coral reef crisis: The critical importance of $<350$ ppm CO2. Marine Pollution Bulletin. 58, p.1428-1436, 2009.

WEIS, V. M. The susceptibility and resilience of corals to thermal stress: adaptation, acclimatization or both? Molecular Ecology, v.19, p.1515-1517, 2010.

WILKINSON, C. Status of coral reefs of the world: 2008.Global Coral Reef Monitoring Network and Reef and Rainforest Research Centre, Townsville, Austrália, 296p. 2008. 